\title{
Outcomes of Femtosecond Laser Assisted Cataract Surgery Performed by Resident and Attending Surgeons
}

\author{
Spencer C. Cleland, MD ${ }^{1}$ Daniel W. Knoch, MD ${ }^{1}$ Jennifer C. Larson, MD ${ }^{1 \odot}$ \\ ${ }^{1}$ Department of Ophthalmology and Visual Sciences, University of \\ Wisconsin-Madison, Madison, Wisconsin \\ J Acad Ophthalmol 2021;13:e26-e31.
Address for correspondence Jennifer C. Larson, MD, Department of Ophthalmology and Visual Sciences, University of Wisconsin- Madison, 2349 Deming Way, Middleton, WI 53562 \\ (e-mail: jciske@wisc.edu).
}

\section{Abstract \\ Keywords \\ - femtosecond-laser assisted cataract surgery \\ - phacoemulsification \\ - resident training \\ - curriculum}

Objective The study aimed to evaluate the safety and efficacy of resident surgeons performing femtosecond laser assisted cataract surgery (FLACS).

Methods A retrospective chart review was conducted at the University of WisconsinMadison from postgraduate year four residents performing FLACS between 2017 and 2019. Data were also collected from residents performing manual cataract surgery, and attending surgeons performing FLACS for comparison. Recorded data included patient demographics, pre- and postoperative visual acuity, pre- and postoperative spherical equivalent, nuclear sclerotic cataract grade, ocular and systemic comorbidities, intraocular lens, duration of surgery, cumulative dissipated energy (CDE), and intraoperative and postoperative complications.

Results A total of 90 cases were reviewed with 30 resident manual cases, 30 resident FLACS cases, and 30 attending FLACS cases. Resident manual ( $25.5 \pm 6.8$ minutes) and resident FLACS (17.5 \pm 7.1 minutes) cases took a significantly longer time to complete compared with attending FLACS cases $(13.6 \pm 4.4$ minutes; $p<0.001)$. There was higher CDE in resident FLACS and resident manual cases compared with attending FLACS cases, but the difference was not statistically significant $(p=0.06)$. Postoperative visual acuity was not statistically different at 1-day and 1-month after surgery among the three groups. Resident FLACS complications, which included one case requiring an intraoperative suture to close the wound, two cases with intraoperative corneal abrasions, two cases with postoperative ocular hypertension, and one case with cystoid macular edema, were not significantly greater than attending FLACS complications $(p=0.30)$.

Conclusion The FLACS performed by resident surgeons had comparable visual acuity outcomes to FLACS performed by attending surgeons, and to manual cataract surgery performed by resident surgeons. However, resident FLACS cases took significantly longer time to complete, and they were associated with a higher CDE and minor complication rate compared with attending FLACS cases. Introducing advanced technologies into surgical training curricula improves resident preparedness for independent practice, and this study suggests FLACS can be incorporated safely and effectively into resident education. received

June 14, 2020 accepted after revision November 18, 2020
DOI https://doi.org/ 10.1055/s-0041-1725582. ISSN 2475-4757.

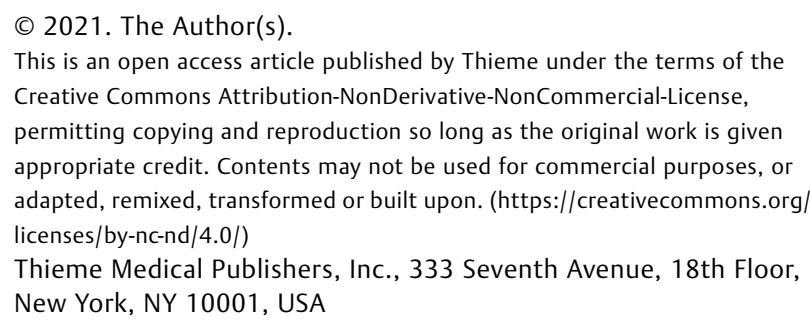


The learning curve for resident surgeons performing cataract extraction with phacoemulsification has been well studied, demonstrating that complication rates decrease with the number of surgeries performed. ${ }^{1,2}$ The Accreditation Council of Graduate Medical Education (ACGME) requires that resident surgeons perform a minimum of 86 cataract surgeries as the primary surgeon, and the national average is $208 \pm 68$ (mean \pm standard deviation) cases among U.S. graduates. ${ }^{3}$ However, the ACGME does not provide guidelines or requirements regarding cataract surgery technique, approach, or the implementation of new technology. ${ }^{4}$

Advancing technology has drastically altered the way cataract surgery is performed with the introduction of phacoemulsification and astigmatism-correcting lenses, which have reduced surgical complications and improved patient outcomes. The femtosecond laser was approved for cataract surgery by the Food and Drug Administration in 2010 , and has gained popularity by offering automation and greater precision to several surgical steps, including corneal wounds, anterior capsulotomy, and lens fragmentation. ${ }^{5-7}$ Although femtosecond laser assisted cataract surgery (FLACS) has been demonstrated to be safe, studies have shown that complications are more common in the first 200 cases performed. $^{7-11}$ This extended learning curve suggests that resident surgeons should be exposed to FLACS under the supervision of an experienced surgeon prior to graduation in attempt to better prepare them to operate safely in future independent practice.

Ophthalmology residency programs are given flexibility to incorporate new technology into their cataract training program, including FLACS and other advanced procedures. However, a survey conducted by Yeu et al reported a perceived deficiency in preparing ophthalmology residents for advanced procedures and techniques that they are likely to perform after completing training. ${ }^{12}$ A 2017 survey of 71 program directors revealed only $44.1 \%$ of residency programs had residents perform FLACS. ${ }^{13}$ The greatest barriers to introducing FLACS to residency training curricula include cost, location of the femtosecond laser, inexperienced faculty, training obstacles, and quality concerns. ${ }^{14}$ In our practice at Madison Surgery Center, FLACS requires an increased out-of-pocket contribution from the patient. While industry sponsored programs help to alleviate some of these costs for resident performed cases, the remaining out of pocket contribution may still be significant for our patients that live on a fixed income. Combined, we find the cost of FLACS, along with limited available outcomes data from the trainee performed procedures, has severely limited our ability to recruit patients appropriate for FLACS who are also willing to have the procedure performed by a trainee.

To begin to address these barriers to expanding our FLACS training program, as well as other advances surgical training programs around the country, we evaluated the safety and efficacy outcomes of resident performed FLACS by comparing these surgeries to attending performed FLACS cases and resident manual cataract extraction cases.

\section{Methods}

After receiving institutional review board approval, a retrospective case-control study of resident- and attending-performed FLACS and resident-performed manual cataract surgery was completed. In our training program, FLACS procedures are performed at the Madison Surgery center (MSC, Madison, WI) by residents in their final year of training during their 4-month comprehensive ophthalmology rotation. Here, we include the data of all FLACS cases performed by senior residents at MSC over the 2017 to 2018 and 2018 to 2019 academic years (six residents and five cases per resident). Our trainees also perform a small number of FLACS procedures at the William S. Middleton Veterans Hospital. Data from those procedures are not included in this report to maintain results that are generalizable to a larger population and due to patient data usage constraints of the institution. Data from the first five consecutive cases performed by each of the six residents during their comprehensive ophthalmology rotations without the femtosecond laser were included as resident manual cases. The first 15 consecutive femtosecond laser assisted cases performed by attending ophthalmologists during the same academic year time periods were included as attending FLACS cases. Cases that received femtosecond-laser assisted wound construction, anterior capsulotomy, and lens fragmentation were included in the study. All femtosecond laser steps were performed with the LenSx Femtosecond laser system (Alcon Laboratories, Inc., Fort Worth, TX).

Preoperative data included patient demographics, visual acuity, spherical equivalent, cataract grade, and ocular comorbidities. Cataract grade was based solely on nuclear sclerotic cataract grade and rounded to the nearest half integer. Intraoperative data included intraocular lens (IOL), cumulative dissipated energy (CDE) as measured on the Alcon Centurion Phacoemulsification Unit (Alcon Laboratories, Inc., Fort Worth, TX), length of surgery, and complications. Length of surgery was defined as the time from incision to completion of a case at the operating microscope and did not include the time at the femtosecond laser or the time transporting the patient. Postoperative data included corneal edema, visual acuity at 1-day and 1-month after surgery, spherical equivalent, and complications.

All data were collected and analyzed by using Microsoft Excel 365. Snellen visual acuities were converted to a logarithm of the minimum angle of resolution (LogMAR) scale for comparison. ${ }^{15}$ The statistical methods used for comparison included Pearson's Chi-square test for categorical values and analysis of variance (ANOVA) for continuous variables. Independent $t$-tests were used to assess means between two groups. Differences were accepted at the $p<0.05$ significance level.

\section{Results}

Cataract cases were reviewed from 90 patients with 30 resident manual cases, 30 resident FLACS cases, and 30 attending FLACS cases. The CDE data were not available in 27 cases. Between the three groups, there were no statistically significant differences among patient demographics, preoperative 
Table 1 Baseline patient characteristics

\begin{tabular}{|c|c|c|c|c|}
\hline & $\begin{array}{l}\text { Resident manual, } n=30 \\
\text { Mean (SD) }\end{array}$ & $\begin{array}{l}\text { Resident FLACS, } n=30 \\
\text { Mean (SD) }\end{array}$ & $\begin{array}{l}\text { Attending FLACS, } n=30 \\
\text { Mean (SD) }\end{array}$ & $p$-Value \\
\hline Cases, $n$ & 30 & 30 & 30 & \\
\hline Sex (\% male) & $43 \%$ & $37 \%$ & $43 \%$ & 0.83 \\
\hline Eye (\% right) & $50 \%$ & $50 \%$ & $43 \%$ & 0.84 \\
\hline Age & $71.8(7.1)$ & $69.5(7.5)$ & $67.5(7.56)$ & 0.09 \\
\hline VA preoperative LogMAR & $0.45(0.24)$ & $0.36(0.29)$ & $0.34(0.24)$ & 0.23 \\
\hline SE preoperative & $-1.9(3.3)$ & $-3.1(4.4)$ & $-3.78(4.27)$ & 0.22 \\
\hline Nuclear sclerotic grade & $2.6(0.51)$ & $2.3(0.6)$ & $1.8(0.6)$ & $<0.001$ \\
\hline $\begin{array}{l}\text { IOL, } n(\%) \\
\text { SN60WF } \\
\text { toric } \\
\text { multifocal }\end{array}$ & $\begin{array}{l}27(90 \%) \\
3(10 \%) \\
0(0 \%)\end{array}$ & $\begin{array}{l}14(47 \%) \\
12(40 \%) \\
4(13 \%)\end{array}$ & $\begin{array}{l}3(10 \%) \\
3(10 \%) \\
24(80 \%)\end{array}$ & $<0.001$ \\
\hline
\end{tabular}

Abbreviations: FLACS, femtosecond laser assisted cataract surgery; IOL, intraocular lens; LogMAR, logarithm of the minimum angle of resolution; SD, standard deviation; SE, spherical equivalent, VA, visual acuity.

visual acuity, or preoperative spherical equivalent (- Table 1). Among the three groups, attending FLACS cases had a significantly lower mean nuclear sclerotic cataract grade $(p<0.001)$. Ocular comorbidities, as summarized in -Supplementry Table S1 (available in online version) were not significantly different among the three group ( $p=0.48$ ).

The IOL selection varied among the three groups $(p<0.001)$; $90 \%$ of the resident manual cases used a monofocal SN60WF lens and 10\% used a toric lens, which contrasts with $90 \%$ of attending FLACS cases using premium lenses (toric or multifocal lens). Fifty-three percent of resident FLACS cases used premium lenses as well.

Operative and postoperative outcomes are summarized in - Figs. 1 and 2. There was a statistically significant difference in procedure duration among the three groups at $25.5 \pm 6.8$ minutes in resident manual cases, $17.6 \pm 7.1$ minutes in resident FLACS cases, and $13.6 \pm 4.4$ minutes in attending cases $(p \leq 0.001)$. When comparing the duration of resident and attending FLACS cases, which did not include time performing the femtosecond laser steps, the difference remained statistically significant $(p=0.02)$. There was a trend toward higher CDE used in resident manual and resident FLACS cases compared with attending FLACS cases; however, the difference was not significant $(p=0.06)$. There were no statistical differences in postoperative visual acuity at 1-day or 1-month after surgery as well. The difference in spherical equivalent at postoperative month was significant between the three groups $(p=0.047)$; however, there was not a significant difference when only comparing the resident- and attending-performed FLACS cases $(p=0.70)$.

Regarding intraoperative complications, one resident FLACS case required sutured wound closure, and two resident FLACS cases sustained corneal abrasions. Postoperatively, two resident FLACS and two attending FLACS cases developed ocular hypertension, and one resident manual case and one resident FLACS case developed cystoid macular edema. There were no

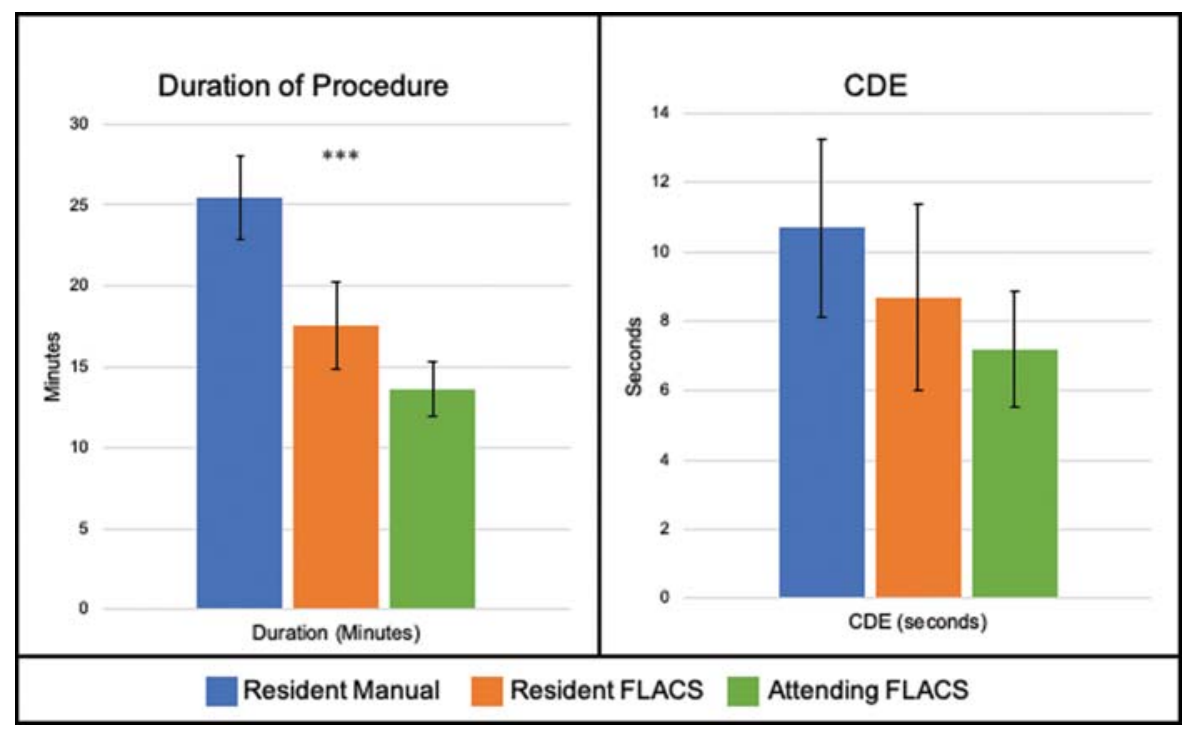

Fig. 1 Operative measures. Duration of the procedure was statistically different $\left(p<0.001^{* * *}\right)$. CDE, cumulative dissipated energy. 


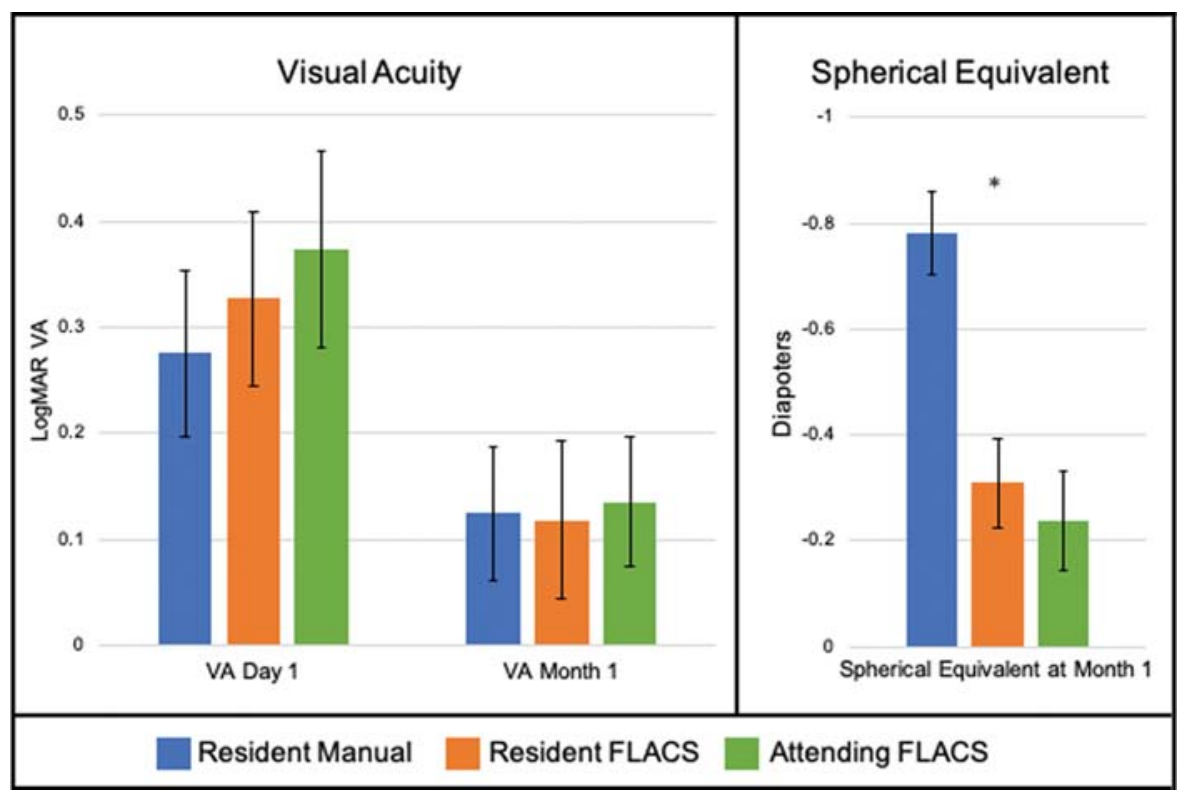

Fig. 2 Postoperative outcomes. Postoperative spherical equivalent was significant comparing resident manual to resident- and attendingperformed FLACS $(p=0.047)$. There was not a significant difference in spherical equivalent when comparing the resident- and attendingperformed FLACS cases $(p=0.70)$. FLACS, femtosecond laser-assisted cataract surgery; LogMAR, logarithm of the minimum angle of resolution; VA: visual acuity.

cases of posterior capsule rupture, anterior vitrectomy, endophthalmitis, or retinal tear/detachment. There was a total complication rate of $3 \%$ in the resident manual cases, $20 \%$ in the resident FLACS cases, and 7\% in the attending FLACS cases, which was not statistically significant $(p=0.30)$.

\section{Discussion}

The femtosecond laser has changed the way many practicing ophthalmologists perform cataract surgery. However, advanced cataract techniques, including FLACS, are perceived as underexposed during residency training, which may be attributed to a lack of ACGME standards or an absence of accepted training models. ${ }^{12}$ Additional barriers to expanding FLACS training for resident surgeons includes cost, lower procedural efficiency, femtosecond-laser location, inexperienced faculty, and quality concerns. ${ }^{14,16}$ Despite these challenges, inclusion of FLACS into training curricula will ease the considerable learning curve for new surgeons who will soon be operating independently. This study examines the safety and efficacy of introducing FLACS into residency surgical curriculum.

Several studies have previously demonstrated longer operating times for both attendings and residents who are beginning to perform FLACS cases compared with traditional phacoemulsification. ${ }^{16-18}$ Similar to Pittner and Sullivan, our data demonstrated that resident cases, performed both manually and with femtosecond laser, and took significantly longer than attending FLACS cases. ${ }^{16}$ Interestingly, our data reveal that duration of resident FLACS cases ( $17.5 \pm 7.1$ minutes) was shorter than resident manual cases ( $25.5 \pm 6.8$ minutes). We attribute this difference in part to how the data were collected; operative duration was measured as the time from start to finish at the operating microscope, which did not include the time at the femtosecond laser or the time transporting the patient from the laser suite to the operating room. The time difference also may be attributed to patients not being signed up for FLACS due to underlying characteristics that may preclude them from being a good candidate for FLACS, such as a small pupil, which would require more time during surgery to address, although this specifically was not evaluated for in this study due to a lack of data regarding preoperative pupil size. Regarding the time difference specifically at the operating microscope, the femtosecond laser created wound, anterior capsulorhexis, and lens fragmentation likely contribute to a shorter operating time in the FLACS cases compared with the traditional cases where these steps were completed manually.

Hou et al showed that resident-performed FLACS cases used 22.5\% less CDE than resident-performed manual phacoemulsification, and lower CDE rates have been correlated with less endothelial cell damage and loss. ${ }^{19}$ Our study revealed a trend toward lower $\mathrm{CDE}$ in resident and attending FLACS cases, although the differences were not statistically significant $(p=0.06)$. The association of lower CDE likely is attributed to the lens fragmentation of the femtosecond laser, requiring less phacoemulsification time needed to sculpt and crack the lens prior to fragment removal.

Postoperative visual acuity among the three groups were comparable postoperatively. However, there was a significant difference in postoperative spherical equivalent between the three groups. This difference is most likely attributed to the higher rate of premium lenses used in resident and attending FLACS cases. Notably, there was not a significant difference in spherical equivalent between resident and attending FLACS cases $(p=0.70)$.

New surgical technology is often accompanied with a learning curve and the potential for an increased number of operative complications, and FLACS is no exception. Bali et 
al and Christy et $\mathrm{al}^{20}$ both describe a learning curve with attending surgeons first using femtosecond laser in their cataract practice. Our study likewise supports this finding; the resident FLACS group had the greatest complication rate at $20 \%$, which was three times greater than the attending FLACS group; however, the difference was not statistically significant $(p=0.30)$. Fortunately, all complications were minor, and none resulted in vision-threatening outcomes. Despite this initial learning curve, our study and other studies demonstrate a similar safety profile and refractive outcomes of FLACS compared with manual cataract surgery supporting its incorporation into resident surgical education. $^{21}$

Although our data were completed by senior residents, Cohen et al proposed integrating the femtosecond laser in residency education as a tool to perform the most complicated steps of cataract surgery. ${ }^{21}$ Doing so could create a step-wise educational model for junior residents allowing them to proceed to the manual steps of cataract surgery after demonstrating competency at the basics of bimanual surgery and surgical steps that were FLACS assisted. Further, earlier integration of the FLACS into residency education may help flatten the learning curve seen with this advanced technology.

Although our analyses yielded statistically significant comparisons between resident FLACS and manual cases and attending FLACS cases, our study was limited by its retrospective design and relatively small sample size. Unfortunately, the sample size was limited by number of available cases, which we see as a symptom of both the relative paucity of data of resident FLACS cases and the absence of an ACGME model or requirement for teaching advanced surgical techniques. A larger sample size would provide a better understanding of FLACS-associated complications rates, which were too low to be significant in our study. In addition, all femtosecond laser steps were performed with the same femtosecond laser machine, which may compromise the generalizability to other femtosecond platforms. Further, the resident FLACS and manual surgery cases were completed by PGY-4 residents who already have a solid foundation in cataract surgery, so our outcomes are limited to this cohort of residents and we cannot comment on the safety and efficacy of resident FLACS in more novice surgeons. Lastly, despite the preoperative data being comparable among all three groups, there was likely selection bias when choosing patients suitable for resident FLACS and manual cases.

\section{Conclusion}

This study provides early evidence of safety, efficacy, and good visual outcomes of resident FLACS, a crucial first step in helping support and build larger resident teaching opportunities in advanced surgical techniques. Resident surgeons performing FLACS were less efficient than attending surgeons at performing FLACS, so additional time should be factored into teaching these cases at academic centers. Introducing FLACS curriculum to residency training presents additional challenges, such as cost and the absence of defined training standards. However, expanding resident surgical curriculum to include FLACS training under the guidance of experienced surgeons will better prepare new graduates to incorporate advanced technologies into their independent practice.

\section{Note}

This study was conducted under the Declaration of Helsinki and approved by institutional review boards at the University of Wisconsin.

\section{Funding}

This study received its financial support from an unrestricted grant of Research to Prevent Blindness, Inc. (New York City, NY).

\section{Conflict of Interest}

None declared.

\section{References}

1 Randleman JB, Wolfe JD, Woodward M, Lynn MJ, Cherwek DH, Srivastava SK. The resident surgeon phacoemulsification learning curve. Arch Ophthalmol 2007;125(09):1215-1219

2 Taravella MJ, Davidson R, Erlanger M, Guiton G, Gregory D. Characterizing the learning curve in phacoemulsification. J Cataract Refract Surg 2011;37(06):1069-1075

3 Accreditation Council for Graduate Medical Education. Review Committee for Ophthalmology. Required Minimum Number of Procedures for Graduating Residents in Ophthalmology. Accessed April 21, 2020 at: https://www.acgme.org/Portals/0/PDFs/ 240_National_Report_Program_Version_2018-2019.pdf

4 Lotfipour M, Rolius R, Lehman EB, Pantanelli SM, Scott IU. Trends in cataract surgery training curricula. J Cataract Refract Surg 2017;43(01):49-53

5 He L, Sheehy K, Culbertson W. Femtosecond laser-assisted cataract surgery. Curr Opin Ophthalmol 2011;22(01):43-52

6 Nagy ZZ. New technology update: femtosecond laser in cataract surgery. Clin Ophthalmol 2014;8:1157-1167

7 Ewe SY, Abell RG, Vote BJ. Femtosecond laser-assisted versus phacoemulsification for cataract extraction and intraocular lens implantation: clinical outcomes review. Curr Opin Ophthalmol 2018;29(01):54-60

8 Nagy ZZ, Mastropasqua L, Knorz MC. The use of femtosecond lasers in cataract surgery: review of the published results with the LenSx system. J Refract Surg 2014;30(11):730-740

9 Bali SJ, Hodge C, Lawless M, Roberts TV, Sutton G. Early experience with the femtosecond laser for cataract surgery. Ophthalmology 2012;119(05):891-899

10 Roberts TV, Lawless M, Bali SJ, Hodge C, Sutton G. Surgical outcomes and safety of femtosecond laser cataract surgery: a prospective study of 1500 consecutive cases. Ophthalmology 2013; 120(02):227-233

11 Brunin G, Khan K, Biggerstaff KS, Wang L, Koch DD, Khandelwal SS Outcomes of femtosecond laser-assisted cataract surgery performed by surgeons-in-training. Graefes Arch Clin Exp Ophthalmol 2017;255(04):805-809

12 Yeu E, Reeves SW, Wang L, Randleman JB, Yeu EASCRS Young Physicians and Residents Clinical Committee. Resident surgical experience with lens and corneal refractive surgery: survey of the ASCRS Young Physicians and Residents Membership. J Cataract Refract Surg 2013;39(02):279-284

13 Yen AJ, Ramanathan S. Advanced cataract learning experience in United States ophthalmology residency programs. J Cataract Refract Surg 2017;43(10):1350-1355 
14 Shah RD, Sullivan BR. Resident training in femtosecond laserassisted cataract surgery: national survey. J Cataract Refract Surg 2015;41(07):1531-1533

15 Tiew S, Lim C, Sivagnanasithiyar T. Using an excel spreadsheet to convert Snellen visual acuity to LogMAR visual acuity. Eye (Lond) 2020;34(11):2148-2149

16 Pittner AC, Sullivan BR. Resident surgeon efficiency in femtosecond laser-assisted cataract surgery. Clin Ophthalmol 2017; 11:291-297

17 Lubahn JG, Donaldson KE, Culbertson WW, Yoo SH. Operating times of experienced cataract surgeons beginning femtosecond laser-assisted cataract surgery. J Cataract Refract Surg 2014;40 (11):1773-1776
18 Chang JSM, Chen IN, Chan WM, Ng JCM, Chan VKC, Law AKP. Initial evaluation of a femtosecond laser system in cataract surgery. J Cataract Refract Surg 2014;40(01):29-36

19 Hou JH, Prickett AL, Cortina MS, Jain S, de la Cruz J. Safety of femtosecond laser-assisted cataract surgery performed by surgeons in training. J Refract Surg 2015;31(01):69-70

20 Christy JS, Nath M, Mouttapa F, Venkatesh R. Learning Curve of femtosecond laser-assisted cataract surgery: Experience of surgeons new to femtosecond laser platform. Indian J Ophthalmol 2017;65(08):638-689

21 Cohen MN, Intili A, Ni N, Blecher MH. Femtosecond laser-assisted cataract surgery in residency training. Curr Opin Ophthalmol 2015;26(01):56-60 